\title{
The correlation between mental health and multiple chemical sensitivity: a survey study in Japanese workers
}

\author{
Xiaoyi Cui $\cdot$ Xi Lu $\cdot$ Aya Hisada $\cdot$ Yuki Fujiwara $\cdot$ \\ Takahiko Katoh
}

Received: 23 September 2014/ Accepted: 27 November 2014/Published online: 11 December 2014

(c) The Japanese Society for Hygiene 2014

\begin{abstract}
Objective This study was designed to determine the correlation between mental health and multiple chemical sensitivity (MCS).

Method The present study was conducted at two companies in 2011; both in Kyushu, Japan. The "subjective symptoms" subscale of the "Self-diagnosis Checklist for Assessment of Workers' Accumulated Fatigue" was used as a mental health subscale. To determine if multiple chemical exposure has an impact on mental health, we composed an original path model using structural equation analysis.

Result Our final path model can be regarded as good: $\mathrm{CMIN} / \mathrm{DF}=1.832, \mathrm{CFI}=0.996$, and RMSEA $=0.038$, $\mathrm{AIC}=71.158$. As expected, chemical sensitivity and other chemical sensitivity scores predicted the health effects of multiple chemical exposure $(\beta=0.19,0.64)$. Mental health was predicted by symptom severity and life impact ( $\beta=0.56$ and 0.12 ), which were both affected by multiple chemical exposure ( $\beta=0.38$ and 0.89 , respectively).

Conclusion As far as we are aware, this is the first study using path analysis to explore whether MCS can indicate mental health in worker populations worldwide, and we found a significant causal relationship between them. This could indicate that more focus should be placed on the impact of MCS on mental health in future investigations.
\end{abstract}

Keywords Mental health · Multiple chemical sensitivity · Other chemical sensitivity · Quick Environmental Exposure and Sensitivity Inventory · Japanese workers

X. Cui $(\bowtie) \cdot X$. Lu $\cdot$ A. Hisada $\cdot$ Y. Fujiwara $\cdot$ T. Katoh Department of Public Health, Faculty of Life Sciences, Kumamoto University, 1-1-1 Honjou, Chuo-ku, Kumamoto 860-8556, Japan e-mail: bjcxy860508@hotmail.com

\section{Introduction}

Multiple chemical sensitivity (MCS) is an acquired chronic dysfunction in which exposure to low levels of chemicals induces mild to severely disabling symptoms [1]. Symptoms are usually vague and nonspecific. In general, the reported symptoms are attributed to previous chemical exposure and recur on subsequent exposure to similar, or structurally unrelated, chemicals at levels normally considered to be nontoxic [2]. The clinical characteristics of MCS patients are usually evaluated using the Environmental Exposure and Sensitivity Inventory, or through interviews that rely on retrospective self-reporting [3]. Miller and Prihoda [4] developed a globally standardized self-administered questionnaire, the Quick Environmental Exposure and Sensitivity Inventory (QEESI), which is designed to assist researchers and doctors in screening, research, and evaluating patients with MCS. QEESI is composed of five items: chemical sensitivity, other chemical sensitivity, symptom severity, life impact, and masking index.

Patients who present with environment-related disorders often experience medically unexplained symptoms, which can be attributed to a variety of environmental causes [5]. It has been suggested that even low levels of toxins can cause neuronal alterations, such as limbic kindling, and comorbidity with mental illnesses is very high $[6,7]$. According to previous studies, MCS patients most frequently complain of symptoms such as loss of energy, difficulty in concentration, depressive feelings, memory disturbances, and fatigue $[8,9]$. In addition, individuals diagnosed with MCS showed a significantly higher level of fatigue than individuals who did not have MCS [10]. As patients experience many psychological symptoms, some researchers believe that MCS may be a syndrome 
associated with a pre-existing psychiatric disorder such as anxiety [11]. In addition, although many physicians are skeptical regarding its association with psychiatric disorders, a report by the UK Health and Safety Executive is less dismissive [2, 12]. Proponents of MCS syndrome believe that the psychiatric disorder is caused by sensitivity to multiple chemical exposure at concentrations that are usually well tolerated and people diagnosed with MCS are polysymptomatic [13]. Skeptics and proponents often contribute to the controversy by claiming that chemical sensitivity is completely psychogenic or completely toxigenic. However, cumulative studies indicate that a multifactorial psychobiological process underlies the condition [14].

Although there is no consistent understanding of the etiology of this condition, there is a need to describe the psychiatric or psychological state of people with chemical sensitivity [8]. The negative effects of chemical exposure are a longstanding part of occupational health in industrialized societies [15]. In addition, such problems tend to arise in industrial workers who are exposed to various chemicals [16]. Psychological health of workers could be assessed using the "subjective symptoms" subscale of the Self-diagnosis Checklist for Assessment of Workers' Accumulated Fatigue questionnaire [17]. Based on the background research described above, we designed this study using the path analysis statistical method to determine if the mental health of workers could be assessed using the QEESI criteria subscales (chemical sensitivity, other chemical sensitivity, symptom severity, and life impact) or other factors (masking index, age, and sex). We aimed to verify if mental health could be inferred from QEESI which is widely used as a screening instrument for MCS, and to determine if mental health could be inferred from MCS. Workers employed in two large companies were selected as participants because workers who are exposed to various chemicals allowed for easier follow-up than community residents, and the response rate may be higher.

\section{Materials and methods}

\section{Study characteristics}

The present study was carried out at a paper pulp producing company and at an automotive company in 2011; both companies were located in Kyushu, Japan. After excluding invalid questionnaires, we obtained 565 valid responses. All study subjects completed the QEESI questionnaire, which also covered age and sex. The characteristics of the entire study population and the distribution of the QEESI subscale and mental health subscale scores are presented in Table 1.
Table 1 Characteristics of the entire study population and distribution of QEESI subscale and mental health subscale scores

QEESI Quick Environmental Exposure and Sensitivity Inventory

Study instruments

The Japanese version of the QEESI questionnaire prepared by Ishikawa and Miyata [18] was used. The subscale for each criterion of the QEESI questionnaire contains ten questions rated on a scale from 0 to 10 ; the total possible score for each subscale ranges from 0 to 100 , with the exception of masking index. As defined by Miller and Prihoda, the criteria for chemical sensitivity and symptom severity are low $=0-19$, medium $=20-39$, and high $=40-100$. The criteria for other chemical sensitivity are low $=0-11$, medium $=12-24$, and high $=25-100$.

Hojo et al. designed a study to establish a new cutoff value for Japanese using the QEESI questionnaire for screening MCS patients. The new cutoff values for 
Japanese subjects were determined to be $\geq 40$ for the "chemical sensitivity" subscale, $\geq 20$ for the "symptom severity" subscale, and $\geq 10$ for the "life impact" subscale. In addition, as the mental health subscale, we used the "subjective symptoms" subscale of the "Self-diagnosis Checklist for Assessment of Workers' Accumulated Fatigue," which contains 13 questions rated on a scale from 0 to 3 . The "subjective symptoms" subscale was categorized into four grades as follows: $0-4$ points as grade I, 5-10 points as grade II, $11-20$ points as grade III, and $\geq 21$ points as grade IV by the Ministry of Health, Labour and Welfare (http://www.mhlw.go.jp/topics/2004/06/dl/ tp0630-1a.pdf).

The chemical sensitivity scale of the survey asked the subjects to list various odors or chemical exposures that made them feel sick such as insecticide, paint or paint thinner, gasoline vapors, fresh tar or asphalt, tobacco smoke, and diesel or gas engine exhaust fumes. The other chemical sensitivity scale contains ten questions concerning exposures other than chemical inhalants that MCS patients frequently describe as making them ill. Included items related to various triggers (chlorinated tap water, foods or food additives, food cravings or feeling ill if a meal is missed, feeling ill after a meal, caffeine sensitivity, caffeine withdrawal symptoms, feeling ill after a small amount of an alcoholic beverage), various skin irritations, medical drugs or devices, and allergens for which classical allergic responses (asthma, nasal symptoms, hives, anaphylaxis, or eczema) have been noted [4]. For the symptom severity scale subjects were asked about symptoms they may have commonly experienced such as muscle or joint problems such as aches and pains, headaches or a feeling of pressure or fullness in the face or head, and skin problems such as a rashes, hives, or dry skin. The life impact scale of the survey asked subjects if they were sensitive to certain chemicals or foods, and if the sensitivities affected any aspects of their life such as diet, the ability to attend work or school, and choice of clothing. The masking index scale was designed to assess the extent to which respondents may have ongoing exposures. The ten questions on masking index (scored yes $=1$ or no $=0$ ) asked subjects whether they regularly used tobacco products, alcoholic beverages, caffeine, certain drugs, or scented personal care products; whether they are exposed to chemicals at work; whether they live with a smoker; whether they use propane or gas for cooking; whether pesticides have been applied at their home or workplace in the past year; and whether they used scented fabric softeners in laundering their clothes or bedding [4].

The mental health scale (scored hardly ever $=0$, sometimes $=1$, or often $=3$ ) questions asked the workers about subjective symptoms they had been aware of in the past month, composed of irritable, anxiety, restless, depression, cannot sleep, feeling ill, lack of concentration, often make mistakes, feeling very sleepy during work, unmotivated, exhaustion, feel tired when waking up in the morning, and get tired more easily than previously.

\section{Statistical analysis}

Anonymous information was collected and used for data analysis. A structural regression (SR) path was composed

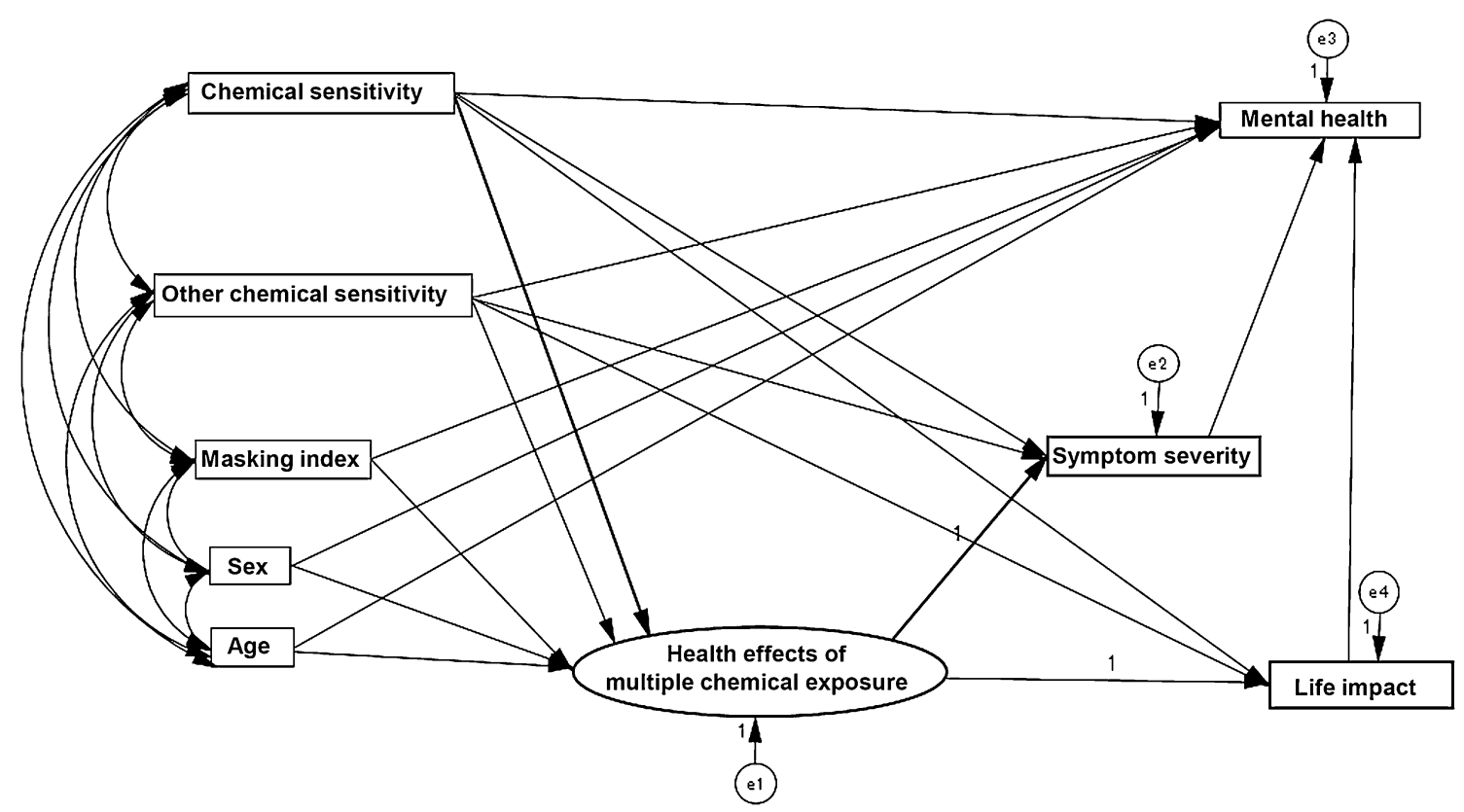

Fig. 1 An original structural regression (SR) path model to estimate correlations between all variables 
using structural equation analysis to examine if chemical exposure had an impact on mental health (Fig. 1). Before constructing the SR model, the means and SDs of the variable scores were calculated and the correlations between them were estimated (Table 3 ).

For the SR model, we created an original path model according to our hypothesis. We set the covariance between the variables of chemical sensitivity, other chemical sensitivity, masking index, and demographics (age and sex). We posited that (1) the health effects of multiple chemical exposure, a latent variable, would be predicted by chemical sensitivity and other chemical sensitivity, and may be affected by masking index, sex and age; (2) symptom severity and life impact would be predicted by the observed variables of chemical sensitivity and other chemical sensitivity, and also by the latent variable of health effects of multiple chemical exposure; and (3) mental health would be predicted by symptom severity and life impact which are both influenced by multiple chemical exposure, and also may be affected by chemical sensitivity, other chemical sensitivity, masking index, sex, and age (Fig. 1).

The fit of the model with the data was examined by $\chi^{2}$ (CMIN), Comparative Fit Index (CFI), Root Mean Square Error of Approximation (RMSEA), and Akaike's Information Criterion (AIC). According to conventional criteria, a good fit is indicated by CMIN/DF $<2$, CFI $>0.97$, and RMSEA $<0.05$, and an acceptable fit by CMIN/DF $<3$, CFI $>0.95$, RMSEA $<0.08$, and a relatively small AIC. All statistical analyses were conducted using SPSS version 21.0 and Amos 21.0.

\section{Ethical statement}

The ethics review board of Kumamoto University (no. 168; May 11, 2011) approved this study in accordance with their ethical guidelines for human research. All participants provided consent, and the complete protection of their personal data was agreed upon in writing.

\section{Results}

The characteristics of the entire study population and the distribution of subscale scores of the QEESI and mental health subscales are shown in Table 1. One hundred and ninety-five $(34.5 \%)$ subjects were over 50 years of age, and there were more males than females ( 93.5 vs. $6.5 \%$ ). The proportion of subjects with high QEESI scores were as follows: chemical sensitivity $(\geq 40,10.1 \%)$, other chemical sensitivity $(\geq 25,5.5 \%)$, symptom severity $(\geq 40,11.3 \%)$, and life impact $(\geq 24,3.0 \%)$. In addition, $8.7 \%$ of the subjects had a high mental health subscale score $(\geq 21)$. The mean mental health score of the MCS patients diagnosed using the QEESI questionnaire with the Miller criteria was higher than that of the controls (22.88 vs. 7.61, $p<0.001$; Table 2). The mean mental health score of the MCS patients diagnosed using the QEESI questionnaire with the Hojo criteria was also higher than that of the controls (18.70 vs. 6.17, $p<0.001$; Table 2).

The correlations between variables and the mean value of each variable used in the path analysis were calculated (Table 3). As expected, the multiple chemical exposure variables of chemical sensitivity (Variable 2) and other chemical sensitivity (Variable 3) were both positively correlated with the health effect variables of symptom severity (Variable $5, R=0.44$ and $0.60, p<0.01$ ), life impact (Variable 6, $R=0.46$ and $0.65, p<0.01$ ), and mental health (Variable 7, $R=0.30$ and $0.38, p<0.01$ ). Mental health (Variable 7) was positively correlated with the health effect variables of symptom severity (Variable 5, $R=0.62, p<0.01$ ) and life impact (Variable 6, $R=0.44$, $p<0.01$ ). The mean age (Variable 1 ) was 43.74 years. The mean scores for the chemical sensitivity, other chemical sensitivity, masking index, symptom severity, life impact, and mental health subscales were 15.18, 7.47, 5.27, 19.30, 4.53, and 9.22, respectively. Masking index (Variable 4) showed a slight negative correlation with chemical sensitivity $(R=-0.10, p<0.05)$. The mean age was higher for
Table 2 Mental health scores of the MCS patients and nonMCS patients

\footnotetext{
QEESI Quick Environmental Exposure and Sensitivity Inventory, $S D$ standard deviation, MCS Multiple Chemical Sensitivity

a Criteria number was defined by QEESI criteria subscales ${ }^{\mathrm{b}} p$ value by the Kruskal-Wallis test
}

\begin{tabular}{|c|c|c|c|c|c|}
\hline $\begin{array}{l}\text { Cutoff value for QEESI } \\
\text { criteria subscales }\end{array}$ & $\begin{array}{l}\text { Criteria } \\
\text { number }^{\mathrm{a}}\end{array}$ & Diagnosis & $\begin{array}{l}n(\%) 565 \\
(100 \%)\end{array}$ & $\begin{array}{l}\text { Mental health } \\
\text { Mean (SD) }\end{array}$ & $p^{\mathrm{b}}$ \\
\hline \multicolumn{6}{|l|}{ Miller criteria } \\
\hline 1. Chemical sensitivity $\geq 40$ & 3 subscales & MCS & $8(1.4)$ & $22.88(12.81)$ & \multirow[t]{3}{*}{$<0.001$} \\
\hline 2. Other chemical sensitivity $\geq 25$ & $1-2$ subscales & Others & 105 (18.6) & $15.09(8.28)$ & \\
\hline 3. Symptom severity $\geq 40$ & 0 subscale & Controls & $452(80.0)$ & $7.61(6.37)$ & \\
\hline \multicolumn{6}{|l|}{ Hojo criteria } \\
\hline 1. Chemical sensitivity $\geq 40$ & 3 subscales & $\mathrm{MCS}^{\mathrm{e}}$ & $23(4.1)$ & $18.70(10.96)$ & \multirow[t]{3}{*}{$<0.001$} \\
\hline 2. Symptom severity $\geq 20$ & $1-2$ subscales & Others & $232(41.1)$ & $12.34(7.80)$ & \\
\hline 3. Life impact $\geq 10$ & 0 subscale & Controls & $310(54.8)$ & $6.17(5.37)$ & \\
\hline
\end{tabular}


Table 3 Pearson correlations between variables and mean values used in the path analysis $(N=565)$

$S D$ standard deviation

$$
\text { Variables }
$$

(1)

Age

(2)

Chemical

(3) Other (4)

(4) (5)

(5)

(6)

(7)

sensitivity

sensitivity index

(1) Age - -

(2) Chemical sensitivity

$0.17^{*}$

(3) Other chemical

$0.010 .54^{* *}$ sensitivity

(4) Masking index

$-0.06-0.10^{*}$

(5) Symptom severity

$0.080 .44^{* * *}$

$-0.02$

(6) Life impact

$-0.01$

$0.46^{* *}$

$0.60^{* *}$

(7) Mental health

$-0.07 \quad 0.30^{* *}$

$0.65^{* * *}$

$-$

Mean

$43.74 \quad 15.18$

$0.38^{* * *}$

0.02

$-$

Standard deviation $( \pm)$

$\pm 10.25 \quad \pm 16.82$

7.47

$0.09^{*}$

$0.56^{* *}$

$0.62^{* *}$

$-$

$\begin{array}{llll}5.27 & 19.3 & 4.53 & 9.22\end{array}$

$\pm 9.26$

$\pm 1.68$

$\pm 16.42 \quad \pm 7.1 \quad \pm 7.63$

males (44.22 \pm 9.88 years old $)$ than females (36.78 \pm 12.78 years old) ( $t$ test, $p=0.001$, not shown in Table 3).

As there were significant correlations between many of the variables examined in this study, we created an original path model (Fig. 1). Based on modification indices, some changes were made to the model. Our final path model can be regarded as good: $\mathrm{CMIN} / \mathrm{DF}=1.832, \mathrm{CFI}=0.996$, RMSEA $=0.038$, and AIC $=71.158$ (Fig. 2). The standardized regression coefficients of the variables obtained using the final path model are shown in Table 4. As expected, scores for chemical sensitivity and other chemical sensitivity predicted the health effects of multiple chemicals exposure $(\beta=0.19,0.64)$. The latent variable of health effects of multiple chemical exposure strongly predicted symptom severity and life impact $(\beta=0.38,0.89)$. Furthermore, symptom severity and life impact, as effects of multiple chemical exposure, predicted mental health $(\beta=0.56,0.12)$. Additionally, significant paths were found from masking index and age to mental health ( $\beta=0.08,-0.12)$; however, these two factors were also associated with chemical sensitivity $(R=-0.10,0.17)$. Paths without statistical significance $(p>0.05)$ are not shown in Fig. 2.

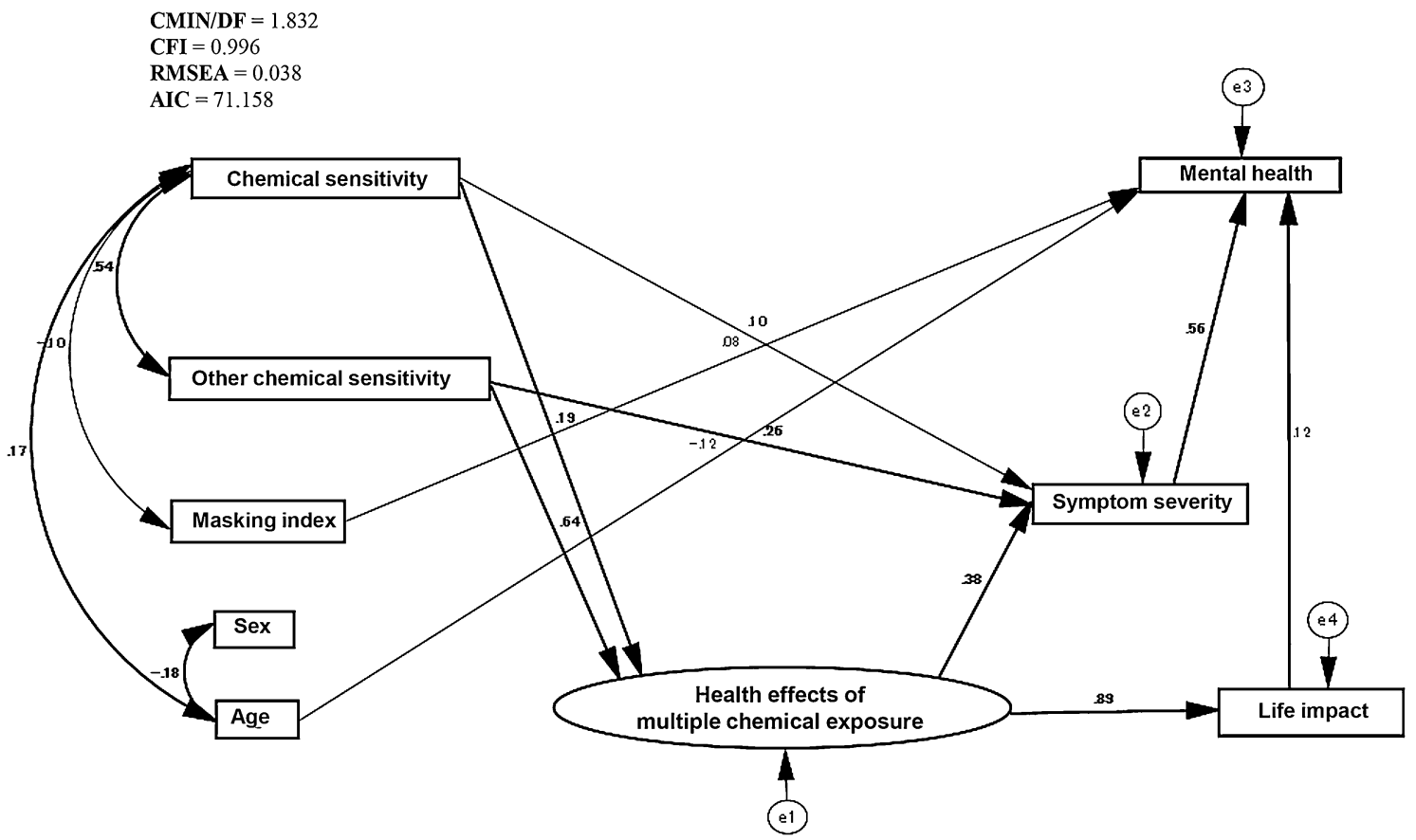

Fig. 2 Final path model: chemical sensitivity and other chemical sensitivity predict health effects of multiple chemical exposure. Health effects of multiple chemical exposure predicts symptom severity and life impact. Mental health is strongly affected by symptom severity and life impact, and slightly affected by age and masking. Path estimates are standardized. Paths without significance $(p>0.05)$ are not shown in the figure (but have not been deleted) 
Table 4 Standardized regression coefficients of variables obtained using the path model $(n=565)$

\begin{tabular}{|c|c|c|c|c|}
\hline Dependent variable & Independent variable & $\beta^{\mathrm{a}}$ & SE & $p^{\mathrm{b}}$ \\
\hline \multirow{5}{*}{$\begin{array}{l}\text { Health impact of multiple } \\
\text { chemical exposure }\end{array}$} & Chemical sensitivity & 0.19 & 0.02 & $* * *$ \\
\hline & Other chemical sensitivity & 0.64 & 0.03 & $* * *$ \\
\hline & Masking index & 0.05 & 0.13 & NS \\
\hline & Age & -0.03 & 0.02 & NS \\
\hline & Sex & 0.01 & 0.90 & NS \\
\hline \multirow[t]{3}{*}{ Symptom } & Chemical sensitivity & 0.10 & 0.04 & $*$ \\
\hline & Other chemical sensitivity & 0.26 & 0.07 & $* * *$ \\
\hline & $\begin{array}{l}\text { Health impact of multiple } \\
\text { chemical exposure }\end{array}$ & 0.38 & - & - \\
\hline Life impact & $\begin{array}{l}\text { Health impact of multiple } \\
\text { chemical exposure }\end{array}$ & 0.89 & - & - \\
\hline \multirow[t]{5}{*}{ Mental health } & Symptom & 0.56 & 0.02 & $* * *$ \\
\hline & Life impact & 0.12 & 0.04 & $* *$ \\
\hline & Age & -0.12 & 0.02 & $* * *$ \\
\hline & Masking index & 0.08 & 0.15 & $*$ \\
\hline & Sex & -0.03 & 1.01 & NS \\
\hline
\end{tabular}

studies [21, 22], MCS patients had higher rates of current mental disorders than controls [3]. In eight investigations, psychiatric disorders were found in $36-100 \%$ of MCS patients [23]. Environmental physicians argue that mental symptoms in MCS patients should be considered as toxic effects of environmental chemicals or a psychological response to a chronic intoxication [24, 25]. Chemical reactions that are triggered in MCS patients have been demonstrated to elicit anxiety attacks, which are associated with objective biological markers such as $\mathrm{PCO}_{2}$ [26]. Lowlevel exposures are sporadic. The dose-duration-response relationship could not be explained [27]. Some researchers suggest that chronic, debilitating illness can induce the biological processes contributing to MCS leading to depression, anxiety, or unexplained physical complaints [13]. In this study, we had no means to measure the doseduration-response effect and the triggered toxic exposure time. Therefore, in this study, the range of time of chemical exposure, time of symptom severity onset, and life impact is specified as the previous year based on the Japanese version of the QEESI questionnaire. The range of time of mental symptom onset is specified as the previous month. This implies that factors associated with symptom severity and life impact that are induced by multiple chemical exposure may occur earlier than mental symptoms.

Treatment of psychiatric symptoms only has not been shown to resolve chemical sensitivity [14]. The QEESI questionnaire is a self-reporting tool that can recognize MCS symptoms and be used in the development of a more effective treatment plan [14]. This tool may help to pinpoint potential environmental chemical, food, and drug contributors, which can lead to improved intervention strategies. Based on the results of this study, notably the strong association between the "other chemical 
sensitivity" scale and "symptom severity" and "mental health" in workers, intervention in individuals with relatively high "other chemical sensitivity" and "mental health" scores could allow them to avoid triggers from items such as chemicals, foods, coffee, alcoholic beverages, and cosmetics.

In addition, we found a mild negative association between masking index and chemical sensitivity and a mild positive association between masking index and mental health. This may be explained by dependence on alcohol or other drugs are very rare diagnosis in patients with environmental illness [5]. Possible explanations for the association with mental health are masking index (smoking, drinking, perfume use, drug dependence, etc.,) related to a true drug sensitization which may induce mental symptoms. It is interesting that a significant difference in age was observed between males and females; this may be explained by sociological reasons for older people in the workplace being mainly male.

In conclusion, this is the first use of path analysis for the exploration of chemical sensitivity-related subscales in the QEESI questionnaire to assess mental health in a Japanese worker population. We found a significant causal relationship between these factors using the path model. This indicates that mental health could be inferred from MCS in Japanese workers. In future investigations, more focus should be placed on the impact of MCS to assess mental health.

Acknowledgments This work was supported in part by Grants-inaid for Research on Health Security Control (H23-Kenki-Ippan-010) from the Ministry of Health, Labour and Welfare of Japan (http:// www.mhlw.go.jp/). We express our sincere appreciation to the staff members of the Department of Public Health, Faculty of Life Sciences, Kumamoto University.

Conflict of interest The authors declare that they have no conflict of interest.

\section{References}

1. Lacour M, Zunder T, Schmidtke K, Vaith P, Scheidt C. Multiple chemical sensitivity syndrome (MCS)-suggestions for an extension of the U.S. MCS-case definition. Int J Hyg Environ Health. 2005;208:141-51.

2. Graveling RA, Pilkington A, George JP, Butler MP, Tannahill SN. A review of multiple chemical sensitivity. Occup Environ Med. 1999;56:73-85.

3. Saito M, Kumano H, Yoshiuchi K, Kokubo N, Ohashi K, et al. Symptom profile of multiple chemical sensitivity in actual life. Psychosom Med. 2005;67:318-25.

4. Miller CS, Prihoda TJ. The environmental exposure and sensitivity inventory (EESI): a standardized approach for measuring chemical intolerances for research and clinical applications. Toxicol Ind Health. 1999;15:370-85.

5. Hausteiner C, Mergeay A, Bornschein S, Zilker T, Förstl H. New aspects of psychiatric morbidity in idiopathic environmental intolerances. J Occup Environ Med. 2006;48:76-82.
6. Bornschein S, Hausteiner C, Zilker T, Förstl H. Psychiatric and somatic disorders and multiple chemical sensitivity (MCS) in 264 'environmental patients'. Psychol Med. 2002;32:1387-94.

7. Stewart DE, Raskin J. Psychiatric assessment of patients with "20th-century disease" ("total allergy syndrome"). CMAJ. 1985;133:1001-6.

8. Tonori H, Aizawa Y, Ojima M, Miyata M, Ishikawa S, Sakabe K. Anxiety and depressive states in multiple chemical sensitivity. Tohoku J Exp Med. 2001;193:115-26.

9. Lax MB, Henneberger PK. Patients with multiple chemical sensitivities in an occupational health clinic: presentation and follow-up. Arch Environ Health. 1995;50:425-31.

10. Jason LA, Taylor RR, Kennedy CL. Chronic fatigue syndrome, fibromyalgia, and multiple chemical sensitivities in a communitybased sample of persons with chronic fatigue syndrome-like symptoms. Psychosom Med. 2000;62:655-63.

11. Staudenmayer H, Selner ME, Selner JC. Adult sequelae of childhood abuse presenting as environmental illness. Ann Allergy. 1993;71:538-46.

12. Reid S. Multiple chemical sensitivity-is the environment really to blame? J R Soc Med. 1999;92:616-9.

13. Black DW, Doebbeling BN, Voelker MD, Clarke WR, Woolson RF, Barrett DH, et al. Multiple chemical sensitivity syndrome: symptom prevalence and risk factors in a military population. Arch Intern Med. 2000;160:1169-76.

14. Katerndahl DA, Bell IR, Palmer RF, Miller CS. Chemical intolerance in primary care settings: prevalence, comorbidity, and outcomes. Ann Fam Med. 2012;10:357-65.

15. Moen BE. Chemical sensitivity and the work place environment: research needs. Psychoneuroendocrinology. 2005;30:1039-42.

16. Ashford NA, Miller CS. Chemical exposures: low levels and high stakes. New York: Van Nostrand Reinold; 1997.

17. Otsuka Y, Tatemaru M. Working hours and psychological health among Japanese restaurant services workers. Int J Psychol Couns. 2010;2:65-71.

18. Ishikawa S, Miyata M. Multiple chemical sensitivity-criteria and test methods for diagnosis. Allergol Immunol. 1999;6:90-8.

19. Bascom R. Multiple chemical sensitivity: a respiratory disorder? Toxicol Ind Health. 1992;8:221-8.

20. Hojo S, Kumano H, Yoshino H, Kakuta K, Ishikawa S. Application of Quick Environment Exposure Sensitivity Inventory (QEESI) for Japanese population: study of reliability and validity of the questionnaire. Toxicol Ind Health. 2003;19:41-9.

21. Fiedler N, Kipen HM, DeLuca J, Kelly-McNeil K, Natelson B. A controlled comparison of multiple chemical sensitivities and chronic fatigue syndrome. Psychosom Med. 1996;58:38-49.

22. Davidoff AL, Fogarty L, Keyl PM. Psychiatric inferences from data on psychologic/psychiatric symptoms in multiple chemical sensitivities syndrome. Arch Environ Health. 2000;55:165-75.

23. Bornschein S, Förstl H, Zilker T. Idiopathic environmental intolerances (formerly multiple chemical sensitivity) psychiatric perspectives. J Intern Med. 2001;250:309-21.

24. Bell IR. White paper: Neuropsychiatric aspects of sensitivity to low-level chemicals: a neural sensitization model. Toxicol Ind Health. 1994;10:277-312.

25. Davidoff AL, Keyl PM. Symptoms and health status in individuals with multiple chemical sensitivities syndrome from four reported sensitizing exposures and a general population comparison group. Arch Environ Health. 1996;51:201-13.

26. Leznoff A. Provocative challenges in patients with multiple chemical sensitivity. J Allergy Clin Immunol. 1997;99:438-42.

27. Staudenmayer H. Idiopathic environmental intolerances (IEI): myth and reality. Toxicol Lett. 2001;120:333-42. 